\title{
Self-assembly of Palm Kernel Oil Wax Esters in Aqueous Media: A Molecular Dynamics Study
}

\author{
Roghayeh Abedi Karjiban ${ }^{1,2}$, Qiu-Yi Huan ${ }^{1}$, Mohd Basyaruddin Abdul Rahman ${ }^{1,2}$, Mahiran Basri ${ }^{1,2} \&$ Bimo Ario Tejo ${ }^{3}$ \\ ${ }^{1}$ Department of Chemistry, Faculty of Science, UPM Serdang, Universiti Putra Malaysia, Selangor, Malaysia \\ ${ }^{2}$ Enzyme and Microbial Technology Research Centre, Faculty of Biotechnology and Biomolecular Sciences, \\ UPM Serdang, Universiti Putra Malaysia, Selangor, Malaysia \\ ${ }^{3}$ Department of Biotechnology and Neuroscience, Faculty of Life Science, Surya University, 15810 Tangerang, \\ Banten, Indonesia
}

Correspondence: Mohd Basyaruddin Abdul Rahman, Department of Chemistry, Faculty of Science, 43400 UPM Serdang, Universiti Putra Malaysia, Selangor, Malaysia. E-mail: basya@upm.edu.my

Received: July 16, 2013 Accepted: September 5, 2013 Online Published: April 3, 2015

doi:10.5539/ijc.v7n1p133 URL: http://dx.doi.org/10.5539/ijc.v7n1p133

\begin{abstract}
Experimentally determined phase diagram of palm kernel oil-based wax esters (PKOEs) nano-emulsion was selected to explore an atomic insight into the self-assembly of PKOEs nano-emulsion using molecular dynamics (MD) simulation technique. A model system including PKOEs molecules and nonionic Tween 80 surfactant in aqueous solution was prepared. We used MD to explore a detail atomic insight into the self-assembly process of and the shape of the micelle formed. MD simulation was performed for $20 \mathrm{~ns}$. MD results involving the radius of gyration, the solvent accessible surface areas, the number of clusters formed, and the moment of inertia analysis revealed that the model prepared showed a stable prolate ellipsoid shape.
\end{abstract}

Keywords: palm kernel oil, wax ester, nano-emulsion, tween80, molecular dynamics simulation

\section{Introduction}

Inflammatory problems are related to skin disorders which can be controlled using non-steroidal anti-inflammatory drugs (NSAIDs) such as ibuprofen and diclofenac (Consola et al., 2007). NSAIDs are widely used as pain killers but they can be toxic if they consumed in high dosages. Oral therapy of NSAIDs, e.g. ibuprofen is limited due to the irritation and the ulceration of the gastro-intestinal mucosa (Al-Saidan, 2004). Therefore, they have been formulated in different topical and transdermal emulsions in order to reduce their side effects (Cho \& Choi, 1998; Adrian et al., 2004; Cevc \& Vierl, 2010). The nano size of nano-emulsions helps significantly solve the penetration problem of drug at stratum corneum (Swarnalatha et al., 2008). Nano-emulsions are dispersion of nano size droplets (20-200 nm) (Solans et al., 2005). Nano-emulsions can't form spontaneously because of the surface tension at oil/water interface, thus an extra energy is usually required to break the bigger droplets to smaller ones (Mason et al., 2006). Currently, unsaturated palm oil-based wax esters (PKOEs) are widely used in the formulation of nano-emulsions for cosmetic and pharmaceutical applications (Salim et al., 2011, 2012). Palm oil-based wax esters are synthesized using enzymatic reactions (Sulaiman et al., 2005). PKOEs are a mixture of different esters which illustrate both lipophilic and hydrophilic properties.

Additionally, theoretical studies of nano-emulsion of palmitate ester have illustrated the potential application of palm oil esters for transdermal delivery of NSAIDs (AbediKarjiban et al., 2012). They selected two different compositions of palmitate ester and Tween 80 surfactant molecules in water with and without diclofenac acid from an experimentally determined phase diagram. They found a prolate-like shape for both systems, while adding the hydrophobic diclofenac produced a more compact structure. Molecular dynamics (MD) simulation technique is used to predict the structure and dynamics of the self-assembled structures for many years. MD can effectively simulate the molecular systems at atomic details using selected force fields based on Newton's second law of motion (Van Gunsteren et al., 2008). In MD, atoms and molecules can interact for a selected period of time and produce an atomic detail of the dynamics of atoms involved. The traditional MD simulation calculations have been extensively carried out to explore the self-assembly process in surfactant molecules such 
as octyl glucoside (Bogusz et al., 2000, 2001), $\mathrm{C}_{12} \mathrm{E}_{4}$ in $\beta$-cyclodextrin (Cunha-Silva \& Teixeira-Dias, 2002), sodium dodecyl sulfate (SDS) (Bruce et al., 2002), and sodium octanoate (DeMoura \& Freitas, 2004, 2005).

Abdul Rahman et al. (2009) reported the MD simulation calculations of palm oil-based esters (POEs) nano-emulsions according to experimental results. They selected a composition of water/palm oil ester/span20 with a ratio of 75:5:20 and carried out MD calculations. They got a spherical micelle in which the palm oil ester molecules were surrounded by nonionic Span20 surfactants. In another study by Abdul Rahman et al. (2010), a model consisted of oleyl oleate ester with a mixture of nonionic Span 20 and Tween 80 molecules was simulated in aqueous solution to find the structural properties of the aggregated structures formed. Their MD simulation results showed a cylindrical shape in which the oleyl oleate/Tween 80 system was more compact compared to the oleyl oleate/Span 20 model. Both Span 20 and Tween 80 are among very commonly used nonionic surfactants in nano-emulsion preparation. Here, a model system containing palm kernel oil-based wax ester and Tween 80 has been explored using all-atom level MD simulations calculations based on our previously reported experimental results (Salim et al., 2012). MD was utilized to give a detail insight into palm kernel oil-based wax esters self-assembly and the shape of the aggregates formed.

\section{Method}

Palm kernel oil-based wax ester (PKOEs) is consisted of oleyl caprylate (7.65\%), oleyl caprate (6.69\%), oleyl laurate $(50.08 \%)$, oleyl myristate $(11.53 \%)$, oleyl palmitate $(3.78 \%)$, oleyl stearate $(1.49 \%)$, oleyl oleate $(11.42 \%)$, and oleyl linoleate (3.15\%) (Gunawan et al., 2005). A spot from the isotropic region of ternary phase diagram with a ratio of 75:5:20 containing Water:PKOEs:Tween80 was chosen to calculate the total number of molecules (Salim et al., 2012). Since the actual number of molecules was very large according to our calculations, in order to save the MD simulation time, the total number of molecules was divided by the lowest number of molecule obtained. Only main esters including oleyl oleate, oleyl myristate and oleyl laurate were selected for MD calculations. The model prepared was consisted of 2 oleyl oleate, 2 oleyl myristate, 10 oleyl laurate, and 30 molecules of Tween 80 .

All molecular structures were geometry optimized using WinGAMESS (Schmidt et al., 1993) by applying Density Functional Theory (DFT) based on Becke's three-parameter hybrid functional method (B3LYP) followed by optimization of the molecules at 6-31G level of theory. MD simulations were performed using GROMACS version 3.3.2 (Lindahl et al., 2001; Van der Spoel et al., 2005) using Optimized Potential for Liquid Simulation-All Atom (OPLS-AA) force field (Jorgensen et al., 1996). The randomly placed starting structure was prepared using Packmol (Martinez \& Martinez, 2003). Later on, the model obtained was placed into a 1000 $\mathrm{nm}^{3}$ cubic simulation box using SPC water model.

Energy minimization was performed using the steepest descent and conjugate gradient techniques and it continued until the energy converged to the maximum force smaller than $50 \mathrm{~kJ} \cdot \mathrm{mol}^{-1} \cdot \mathrm{nm}^{-1}$. The minimized model was then underwent $1 \mathrm{~ns}$ of pre-equilibration using isobaric-isothermal ensemble (NPT) followed by a $20 \mathrm{~ns}$ of MD production simulation in a canonical ensemble (NVT). The integrating timestep was set at $2 \mathrm{fs}$ and periodic boundary condition was applied. Temperature kept constant at $300 \mathrm{~K}$ using Berendsen coupling while the pressure turned off during production simulation (Berendsen et al., 1984). Particle Mesh Ewald (PME) method was used to control the electrostatics interactions with a cut-off value of $0.9 \mathrm{~nm}$ (Essman et al., 1995). The cut-off value for van der Waals (VDW) short range forces was also set at $0.9 \mathrm{~nm}$ in order to correlate with electrostatic interactions calculations computed during neighbour searching. The coupling time was $0.1 \mathrm{ps}$ and all-bonds were constraints using LINCS algorithm (Hess et al., 1998).

\section{Results and Discussion}

Emulsions exhibit a size distribution rather than a fixed size at equilibrium (Maillet et al., 1999; Konidala et al., 2006). The aggregation of the Tween 80 and PKOEs molecules can be further explained using radius of gyration $\left(\mathrm{R}_{\mathrm{g}}\right)$ results to find the compactness of the aggregates obtained. Figure 1 shows the fluctuation of $\mathrm{R}_{\mathrm{g}}$ as a function of simulation time. From Figure 1, at $0 \mathrm{~ns}$, the total $R_{g}$ of the system was $3.92 \pm 0.05 \mathrm{~nm}$ and it reduced to $3.08 \pm 0.10 \mathrm{~nm}$ at the end of simulation. However, the biggest compactness was detected from 16 to $17 \mathrm{~ns}$ with an average value of $2.49 \pm 0.05 \mathrm{~nm}$. The big fluctuations from $10 \mathrm{~ns}$ onward until the end of simulation could be as a result of periodic boundary condition.

We calculated the size of the aggregate formed by computing the average diameter of the aggregate at the end of the simulation using VEGAZZ program (Pedretti et al., 2004). The average diameter of the aggregate formed was $5.78 \pm 0.05 \mathrm{~nm}$ compared to which it was approximately consistent with experimentally measured value of $6.03 \pm 0.05 \mathrm{~nm}$ (Salim et al., 2012). The snapshot pictures of PKOEs and Tween 80 surfactant molecules in the simulation box are shown in Figure 2(a) to Figure 2(e). The water molecules were removed from pictures for 
simplicity. From Figure 2(b), the aggregation of molecules was clearly started at $5 \mathrm{~ns}$ and it continued until one big cluster and one smaller cluster were detected at the end of simulation (see Figure 2(e)).

From Figure 1, at $0 \mathrm{~ns}$, the total $\mathrm{R}_{\mathrm{g}}$ of the system was $3.92 \pm 0.05 \mathrm{~nm}$ and it reduced to $3.08 \pm 0.10 \mathrm{~nm}$ at the end of simulation. However, the biggest compactness was detected from 16 to $17 \mathrm{~ns}$ with an average value of $2.49 \pm 0.05$ $\mathrm{nm}$. The big fluctuations from $10 \mathrm{~ns}$ onward until the end of simulation could be as a result of periodic boundary condition. We calculated the size of the aggregate formed by computing the average diameter of the aggregate at the end of the simulation using VEGAZZ program (Pedretti et al., 2004). The average diameter of the aggregate formed was $5.78 \pm 0.05 \mathrm{~nm}$ compared to which it was approximately consistent with experimentally measured value of $6.03 \pm 0.05 \mathrm{~nm}$ (Salim et al., 2012). The snapshot pictures of PKOEs and Tween80 surfactant molecules in the simulation box are shown in Figure 2(a) to Figure 2(e). The water molecules were removed from pictures for simplicity. From Figure 2(b), the aggregation of molecules was clearly started at $5 \mathrm{~ns}$ and it continued until one big cluster and one smaller cluster were detected at the end of simulation (see Figure 2(e)).

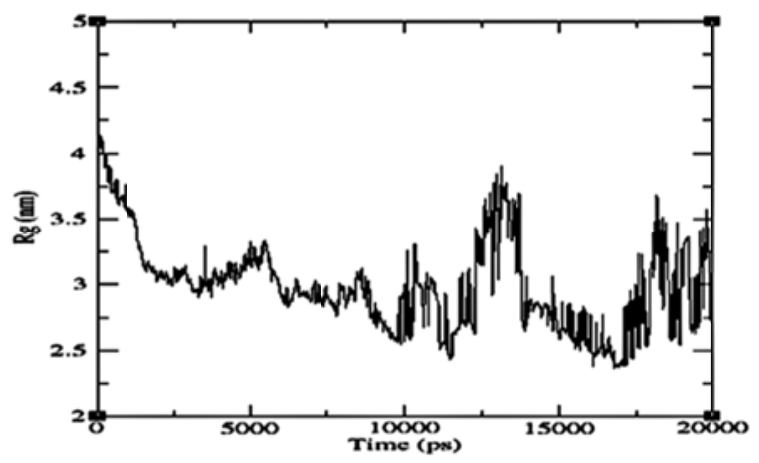

Figure 1. The radius of gyration obtained, with a fluctuation as a function of time for MD simulation of the model PKOEs/Tween80/Water system

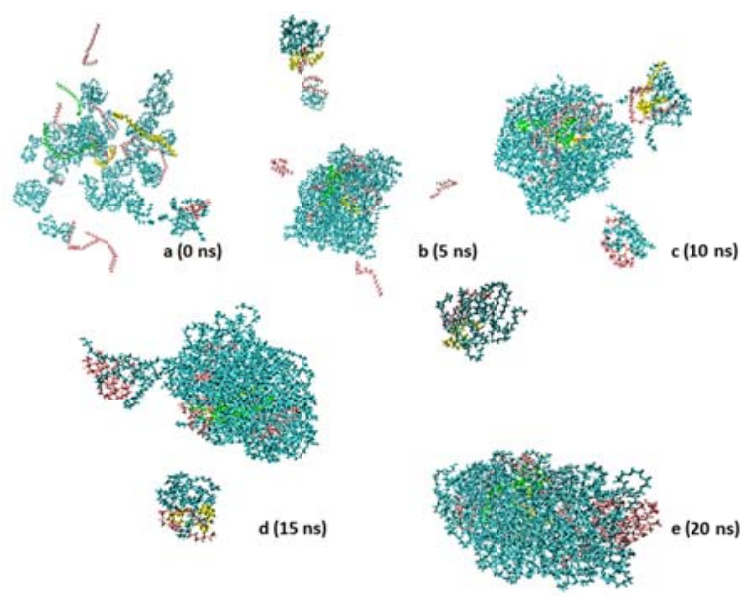

Figure 2. Snapshot pictures during molecular dynamics (MD) simulations of the model PKOEs/Tween80/Water system at 300K; Tween80 (cyan); oleyl laureate (pink); oleyl oleate (green); oleyl myristate (yellow)

The formation of two clusters was confirmed using clustering analysis. g_clustsize analysis tool in GROMACS version 3.3.2 was used to carry out the clustering analysis. The number of clusters was reported based on the distance between surfactant molecules. Molecules that were over $0.5 \mathrm{~nm}$ away from each other were not classified as one cluster. Additionally, we ignored the image as a result of using periodic boundary conditions. Figure 3 shows the number of cluster formed as a function of time. From Figure 3, the model was consisted of 13 clusters when the simulation started. The number of clusters fluctuated drastically between 0 ns and $\sim 3 \mathrm{~ns}$ until the number of clusters was decreased to five and it continued to decreasing to three at $\sim 11 \mathrm{~ns}$. At $14.5 \mathrm{~ns}$, the number of clusters in the simulated model system was two and it remained constant until 20 ns. Therefore, a decrease in the number of clusters formed resulted in an increase of the cluster size.

The shape of the self-assembled structure formed was characterized by using the moment of inertia analysis. The moment of inertia can be computed from the radius of gyration results using Equation (1), where $m_{i}$ is the mass of the atom $i$ and $r_{i}$ is the distance between the atom $i$ and the centre of mass of each molecule; 


$$
\mathrm{R}_{\mathrm{g}}=\left(\frac{\sum_{i} m_{i} r_{i}^{2}}{\sum_{i} m_{i}}\right)^{1 / 2}
$$

The approximate shape evolution over time was detected by calculating the three time-averaged principle moment of inertia ratio $\left(\mathrm{I}_{1} / \mathrm{I}_{3}, \mathrm{I}_{2} / \mathrm{I}_{3}\right.$, and $\left.\mathrm{I}_{1} / \mathrm{I}_{2}\right)$ along the three principle axes $(\mathrm{x}, \mathrm{y}, \mathrm{z})$. The analysis of the moment of inertia ratio is particularly important when a significant deviation from the spherical/symmetrical micellar shapes observes (Gao X, Wong TC, 2000; Kuhn et al., 2002; Tieleman et al., 2006). From our results, the three time-averaged values of the moment of inertia were 1.87:1.59:1.19. The far lowest principal moment of inertia ratio of 1.19 could suggest that the shape of the aggregate formed was a prolate ellipsoid. The results obtained were also consistent with the visual representations showed in Figure 2).

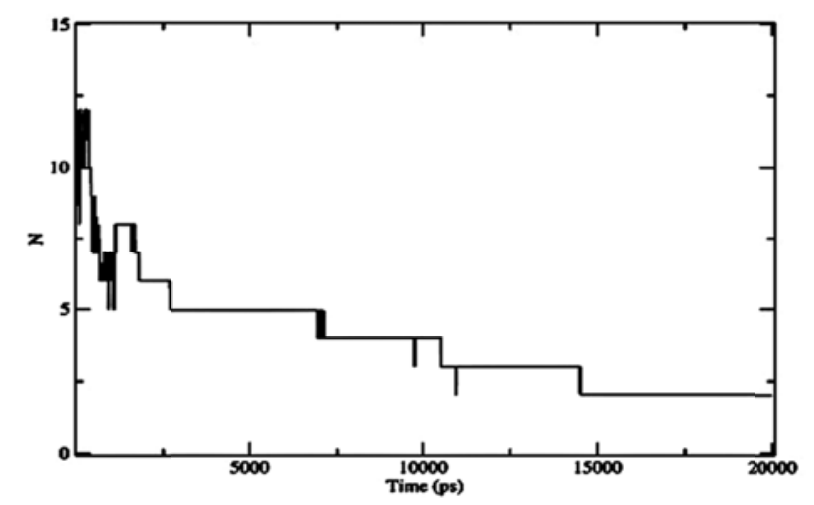

Figure 3. The number of cluster formed, with a fluctuation as a function of time for MD simulation of the model PKOEs/Tween80/Water system

The amphiphilic properties of PKOEs are very important in the forming of aggregates. These amphiphilic properties can execute strong interactions among the hydrophobic tail of the esters (Pfitser \& Helsh, 1999). Furthermore, the oil uptake process with pulling some of the esters from oil droplet could trigger the solubilization of surfactant molecules (Rusling \& Kumosinski, 1995). The formation of aggregates can be further explained by the Ostwald Ripening phenomenon (Weiss et al., 2000; Ratke \& Voorhees, 2002). The molecules which located at the surface of the smaller clusters can be separated and form free monomers. These free monomers will then attach together repeatedly until a stable aggregate forms. The aggregation of molecules to form micelles is due to both hydrophobic and hydrophilic effects (Israelachvili, 1991; Block, 2001). The model system, palm kernel oil-based esters and Tween80, with a high hydrophilic-lipophilic balance (HLB) value of $\sim 15$ can assist the formation of a stable self-assembled structure. Accessible surface area (ASA) is considered as a good approach to gain a quantitative interpretation of molecular packing. The water accessibility of surfactants can be evaluated by computing the solvent accessible surface area (SASA). The SASA per each molecule of surfactant is usually dependent on the micelle size.

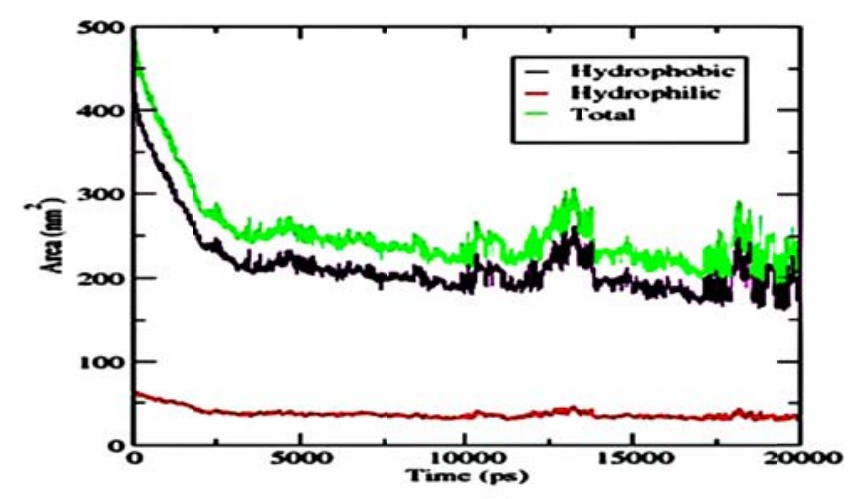

Figure 4. The solvent accessible surface area (SASA), with a fluctuation as a function of time for MD production simulation of the model PKOEs/Tween80/Water system 
Figure 4 shows the solvent accessible surface area (SASA) fluctuation as a function of time. The SASA analysis was done by using g_sas tool in GROMACS version 3.3.2 which computes hydrophobic, hydrophilic and total solvent accessible surface area. The average initial values of the hydrophobic, hydrophilic and the total SASA were $427.30 \pm 3.80 \mathrm{~nm}^{2}, 64.20 \pm 1.20 \mathrm{~nm}^{2}$, and $491.40 \pm 3.80 \mathrm{~nm}^{2}$, respectively. A drastic decrease was observed for the hydrophobic and the total SASA until 3 ns and then it remained more or less constant until the end of simulation. The minimum value of SASA was detected around $\sim 18 \mathrm{~ns}$ with an average value of $171.00 \pm 1.20 \mathrm{~nm}^{2}$ for the hydrophobic part and $202.40 \pm 1.20 \mathrm{~nm}^{2}$ for the total accessibility where the most compact aggregate was detected. Our SASA results may propose that the self-assembly of the PKOEs esters and Tween 80 surfactant molecules could be mainly due to the hydrophobic interaction resulting in from the amphiphilic characteristics of both esters and nonionic surfactant molecules.

\section{Conclusion}

Palm kernel oil-based nano-emulsion formulation can be a suitable alternative for transdermal delivery of active ingredients such as NSAIDs. These kinds of formulations can be used as carriers for the delivery of NSAIDs by removing the adverse effects of drug due to the oral therapy showed experimentally (Salim et al., 2011, 2012). We performed all-atom level MD simulations of the model Water, PKOEs, and Tween 80 provided by our experimental groups to explore how can the self-assembly of nano-emulsions containing palm kernel oil wax esters and nonionic Tween 80 surfactants proceed in aqueous solution and what is the shape of aggregate formed. Our structural analysis showed a stable prolate ellipsoid shape at the end of MD simulation. From our results, it can clearly be seen that the self-assembly of the esters and surfactants might be mainly due to the hydrophobic interaction due to the amphiphilic characteristics of both esters and surfactant molecules.

\section{Acknowledgements}

The authors gratefully acknowledge financial support from GRF UPM, and RUGS 9342300.

\section{References}

Abdul Rahman, M. B., Huan, Q-Y, Tejo, B. A., Basri, M., Salleh, A. B., \& Abdul Rahman, R. N. Z. (2009). Self-assembly formation of palm-based esters nano-emulsion: A molecular dynamics study. Chem. Phys. Lett., 36, 220-224. http://dx.doi.org/10.1016/j.cplett.2009.08.073

Abdul Rahman, M. B., Latif, M. A. M., Basri, M., Salleh, A. B., \& Abdul Rahman, R. N. Z. (2010). Molecular dynamics simulation of oleyl oleate swollen micelles system. Mol. Simul., 36, 403-407. http://dx.doi.org/10.1080/08927020903544535

Abedikarjiban, R., Basri, M., Abdul Rahman, M. B., \& Salleh, A. B. (2012). Molecular dynamics simulation of palmitate ester self-assembly with diclofenac. Int. J. Mol. Sci., 13, 9572-9583. http://dx.doi.org/10.3390/ijms13089572

Adrian, P. F., Schiller, R., Motzkus, H. W., Gunther, C., Muller, R. H., \& Lipp, R. (2004). Transdermal Delivery of Highly Lipophilic Drugs: In Vitro Fluxes of Antiestrogens, Permeation Enhancers, and Solvents from Liquid Formulations. Pharm. Res., 19, 661-668.

Al-Saidan, S. M. (2004). Transdermal self-permeation enhancement of Ibuprofen. J. Controlled Release, 100, 199-209. http://dx.doi.org/10.1016/j.jconrel.2004.08.011

Block, S. S. (2001). Surface-Active Agent In: Disinfections, sterilization and preservation. Philadelphia: Lippincott William and Wilkins.

Berendsen, H. J. C., Postma, J. P. M., van Gunsteren, W. F., DiNola, A., \& Haak, J. R. (1984). Molecular dynamics with coupling to an external bath. J. Chem. Phys., 8, 3684-3690. http://dx.doi.org/10.1063/1.448118

Bogusz, S., Venable, R. M., \& Pastor, R. W. (2000). Molecular dynamics simulations of Octyl Glucoside Micelles: Structural properties. J. Phys. Chem. B, 104, 5462-5470. http://dx.doi.org/10.1021/jp000159y

Bogusz, S., Venable, R. M., \& Pastor, R. W. (2001). Molecular dynamics simulations of Octyl Glucoside Micelles: Dynamic properties. J. Phys. Chem. B, 105, 8312-8321. http://dx.doi.org/10.1021/jp004475o

Bruce, C. D., Berkowitz, M. L., Perera, L., \& Forbes, M. D. E. (2002). Molecular dynamics simulation of sodium dodecyl sulfate micelle in water: Micellar structural characteristics and counterion distribution. $J$. Phys. Chem. B, 106, 3788-3793. http://dx.doi.org/10.1021/jp013616z

Cassidy, J., \& Schätzlein, A. G. (2004). Tumour-targeted drug and gene delivery: principles and concepts. Expert Rev. Mol. Med., 19, 1-17. 
Cevc, G., \& Vierl, U. (2010). Nanotechnology and the transdermal route: A state of the art review and critical appraisal. J. Controlled Release, 141, 277-299. http://dx.doi.org/10.1016/j.jconrel.2009.10.016

Cho, Y-J, \& Choi, H-K (1998). Enhancement of percutaneous absorption of ketoprofen: Effect of vehicles and adhesive matrix. Int. J. Pharm., 169, 95-104. http://dx.doi.org/10.1016/S0378-5173(98)00115-X

Consola, S., Blanzat, M., Perez, E., Garrigues, J-C, Bordat, P., \& Rico-Lattes. I. (2007). Design of original bioactive formulations based on sugar surfactant/nonsteroidal anti-inflammatory catanionic self-assemblies: A new way of dermal drug delivery. Chem. Eur. J., 13, 3039-3047. http://dx.doi.org/10.1002/chem.200601449

Cunha-Silva, L., \& Teixeira-Dias, J. J. C. (2002). Aqueous solution inclusion of the nonionic surfactant C12E4 in $\beta$-cyclodextrin: Implications of micellization in stoichiometry determination and model calculations. J. Incl. Phenom. Macrocycl. Chem., 43, 127-131.

DeMoura, A. F., \& Freitas, L. C. G. (2004). Molecular dynamics Simulation of the sodium octanoate micelle in aqueous solution: Comparison of force field parameters and molecular topology effects on the micellar structure. Braz. J. Phys., 34, 64-72. http://dx.doi.org/10.1590/S0103-97332004000100010

DeMoura, A. F., \& Freitas, L. C. G. (2005). Molecular dynamics simulation of the sodium octanoate micelle in aqueous solution. Chem. Phys. Lett., 411, 474-478. http://dx.doi.org/10.1016/j.cplett.2005.05.039

Essman, U., Perera, L., Berkowitz, M., Darden, T., Lee, H., Pedersen, L.G.(1995). A smooth particle mesh Ewald method. J. Chem. Phys., 103,8577-8593. http://dx.doi.org/10.1063/1.470117

Gao, X., \& Wong, T. C. (2001). Molecular dynamics simulation of adrenocorticotropin (1-10) peptide in a solvated dodecylphosphocholine micelle. Biopolymers, 58, 643-659. http://dx.doi.org/10.1002/1097-0282(200106)58:7<643::AID-BIP1037>3.0.CO;2-R

Gunawan, E. R., Basri, M., Rahman, M. B. A., Salleh, A. B., \& Rahman, R. N. Z. A. (2005). Study on response surface methodology (RSM) of lipase-catalyzed synthesis of palm-based wax ester. Enzyme Microb. Technol., 37, 739-744. http://dx.doi.org/10.1016/j.enzmictec.2005.04.010

Hess, B., Bekker, H., Berendsen, H. J. C., \& Fraaije, J. G. E. M. (1998). LINCS: A linear constraint solver for $\begin{array}{llllll}\text { molecular simulation. } \quad J . & \text { Comput. } & \text { Chem., } & 18, & 1463-1472 .\end{array}$ http://dx.doi.org/10.1002/(SICI)1096-987X(199709)18:12<1463::AID-JCC4>3.0.CO;2-H

Israelachvili, J. N. (1992). Intermolecular and surface forces. London: Academic press.

Jorgensen, W. L., Maxwell, D. S., \& Tirado-Rives, J. (1996). Development and testing of the OPLS all-atom force field on conformational energetics and properties of organic liquids. J. Am. Chem. Soc., 118, 11225-11236. http://dx.doi.org/10.1021/ja9621760

Kuhn, H., Breitzke, B., \& Rehage, H. (2002). A molecular modelling study of pentanol solubilized in a sodium octanoate micelle. J. Colloid. Interface Sci., 249, 152-161. http://dx.doi.org/10.1006/jcis.2001.8149

Konidala, P., He, L., \& Niemeyer, B. (2006). Molecular dynamics characterization of n-octyl-b-D-glucopyranoside micelle structure in aqueous solution. J. Mol. Graphics Modell., 25, 77-86. http://dx.doi.org/10.1016/j.jmgm.2005.11.008

Lindahl, E., Hess, B., \& van der Spoel, D. (2001). GROMACS 3.0: A package for molecular simulation and trajectory analysis. J. Mol. Model., 7, 306-317.

Maillet, J. B., Lachet, V., \& Coveney, P. V. (1999). Large scale molecular dynamics simulation of self-assembly processes in short and long chain cationic surfactants. Phys. Chem. Chem. Phys., 1, 5277-5290. http://dx.doi.org/10.1039/a905216j

Mason, T. G., Wilking, J. N., Meleson, K., Chang, C. B., \& Graves, S. M. (2006). Nanoemulsion: Formation, Structure and Physical Properties. J. Phys. Condens. Matter., 18, 635-666. http://dx.doi.org/10.1088/0953-8984/18/41/R01

Martinez, J. M., \& Martinez, L. (2003). Packing optimization for automated generation of complex system's initial configurations for molecular dynamics and docking. J. Comput. Chem., 24, 819-825. http://dx.doi.org/10.1002/jcc.10216

Pedretti, A., Villa, L., \& Vistoli, G. (2004). Vega-An open platform to develop chemo-bio-informatics applications, using plug-in architehture and script programming. J. Comput-Aided Mol. Des., 18, 167-173. http://dx.doi.org/10.1023/B:JCAM.0000035186.90683.f2 
Pfitser, W. R., \& Helsh, D. S. T. (1990). Permeation enhancers compatible with transdermal drug delivery system: Part 1 Selection and formulation considerations. Pharm. Technol., 14, 52-60.

Rao, S. C., Schoenwald, R. D., Barfknecht, C. F., \& Laban, S. L. (1992). Biopharmaceutical evaluation of ibufenac, ibuprofen, and their hyroxyethoxy analogs in the rabbit eye. J. Pharmacokinet. Biopharm., 20, 357-388. http://dx.doi.org/10.1007/BF01062463

Ratke, L., \& Voorhees, P. W. (2002). Growth and Coarsening: Ostwald Ripening in Material Processing. Berlin: Springer-Verlag. http://dx.doi.org/10.1007/978-3-662-04884-9

Rusling, J. F., \& Kumosinski, T. F. (1995). An approximation to hydrophobic attraction for molecular dynamics of self-assembled surfactant aggregates. J. Phys. Chem., 99, 9241-9247. http://dx.doi.org/10.1021/j100022a043

Salim, N., Basri, M., Abdul Rahman, M. B., Abdullah, D. K., Basri, H., \& Salleh, A. B. (2011). Phase Behaviour, Formation and Characterization of Palm-Based Esters Nanoemulsion Formulation containing Ibuprofen. $J$. Nanomedic. Nanotechnol., 2, 2157-7439. http://dx.doi.org/10.4172/2157-7439.1000113

Salim, N., Basri, M., Abdul Rahman, M. B., Abdullah, D. K., \& Basri, H. (2012). Modification of palm kernel oil esters nanoemulsions with hydrocolloid gum for enhanced topical delivery of ibuprofen. Int. J. Nanomedicine, 7, 4739-4747. PMid:22973096.

Schmidt, M. W., Baldridge, K. K., Boatz, J. A., Elbert, S. T., Gordon, M. S., Jensen, J. H., Koseki, S., Matsunaga, N., Nguyen, K. A., \& Su, S. J. (1993). General atomic and molecular electronic structure system. J. Comput. Chem., 14, 1347-1363. http://dx.doi.org/10.1002/jcc.540141112

Solans, C., Izquierdo, P., Nolla, J., Azemar, N., \& Garcia-Celma, M. J. (2005). Nano-emulsions. Curr. Opin. Colloid Interface Sci., 10, 102-110. http://dx.doi.org/10.1016/j.cocis.2005.06.004

Sulaiman, A., Basri, M., Salleh, A. B., Rahman, R. N. Z., \& Ahmad, R. S. (2005). Phase behavior of oleyl oleate with nonionic surfactants. J. Dispersion Sci. Technol., 26, 1-3. http://dx.doi.org/10.1081/DIS-200062937

Tieleman, D. P., van der Spoel, D., \& Berendsen, H. J. C. (2000). Molecular dynamics simulations of dodecylphosphocholine micelles at three different aggregate sizes: micellar structure and chain relaxation. $J$. Phys. Chem. B., 104, 6380-6388. http://dx.doi.org/10.1021/jp001268f

Van der Spoel, D., Lindahl, E., Hess, B., Groenhof, G., Mark, A. E., \& Berendsen, H. J. C. (2005). GROMACS: Fast, flexible and free. J. Comput. Chem., 26, 1701-1718. http://dx.doi.org/10.1002/jcc.20291

Van Gunsteren, W. S., Bakowies, D., Baron, R., Chandrasekhar, I., Christen, M., Daura, X., Gee, P., Geerke, D. P., Glattli, A., \& Hunenberger, P. H. (2006). Biomolecular modeling: Goals, problems, perspectives. Angew. Chem. Int. Ed., 45, 4064-4092. http://dx.doi.org/10.1002/anie.200502655

Weiss, J., Canceliere, C., \& McClements, D. J. (2000). Mass transport phenomena in oil-in-water emulsions containing surfactant micelle: Ostwald ripening. Langmuir, 16, 6833-6838. http://dx.doi.org/10.1021/la991477v

\section{Copyrights}

Copyright for this article is retained by the author(s), with first publication rights granted to the journal.

This is an open-access article distributed under the terms and conditions of the Creative Commons Attribution license (http://creativecommons.org/licenses/by/3.0/). 